\title{
El palacio urbano: formación de un modelo en la Edad Media
}

\author{
Jean PASSINI \\ (UMR 8558 CNRS-EHESS) \\ jean.passini@ehess.fr
}

\section{RESUMEN}

La pervivencia de estructuras abovedadas medievales en edificaciones posteriores nos muestra las raíces del nuevo modelo de palacio que se desarrollará en el Renacimiento. A través de dos conjuntos palaciegos toledanos: el Palacio de don Manuel y el Palacio de Fuensalida, se presentan los elementos que conformarán la nueva tipología, identificando su origen y analizando sus transformaciones.

Palabras clave: Arquitectura medieval, Toledo, Palacio de Fuensalida, Palacio de don Manuel.

\section{The urban palace: formation of a pattern model in the Middle Ages}

\begin{abstract}
The survival of medieval vaulted structures in later buildings shows us the roots of the palatial new model to be developed in the Renaissance. Through two Toledo palaces complexes: the don Manuel's Palace and the Palace of Fuensalida, we will analyze the elements that will make up the new typology, identify its origin and analyze their transformations.
\end{abstract}

Key words: Medieval architecture, Toledo, The Fuensalida Palace, The don Manuel's Palace. 


\section{Introducción}

Desde que empezó la construcción de la catedral en Toledo, en 1226, hasta principios del s. XVI, la ciudad tuvo un importante desarrollo arquitectónico con la construcción de destacadas obras en las que se introdujeron una serie de elementos nuevos y se transformaron otros que seguían empleándose. Nos proponemos subrayar algunas de las innovaciones que contribuyeron a la formación de un modelo de palacio urbano, teniendo en cuenta las excavaciones arqueológicas y los levantamientos realizados en los últimos veinte años.

Durante cuatro siglos la cultura musulmana, la judía y la cristiana convivieron en tolerancia pero con diferentes reglas que permitieron desarrollar a cada una sus propias formas arquitectónicas. La ciudad de Toledo fue un lugar privilegiado donde se mezclaron esas prácticas constructivas y emergieron nuevas ideas, otorgando a la arquitectura un destacado papel lleno de contenido social.

En los s. XIII y XIV, la ciudad conoció periodos de intensa actividad constructiva: desde la transformación de mezquitas en iglesias, como la parroquia de San Antolín, a la nueva construcción de Santiago del Arrabal, del palacio arzobispal, de hospitales y de construcciones civiles, como las casas principales o los palacios de la familia real. Principalmente, fue un encuentro entre dos formas de hacer, de un lado la de los mudéjares que actuaban desde hacía tiempo en Toledo y, del otro, la de los pedreros cristianos, llegados de fuera para construir la Catedral.

En la Edad Media, cuando una familia decidía construir una casa grande o principal, sus motivaciones, las ideas que inspiraban el proyecto inicial del edificio y los modos de construcción los determinaban directamente el poder y la ciudad. Una casa mayor o palacio era, en sí mismo, una manifestación de poder. En el marco de nuestra investigación, hasta ahora, hemos estudiado dos aspectos en el desarrollo de las casas y casas grandes medievales: primero, la identificación de las dos partes principales que conformaban los conjuntos palaciegos: el palacio, como sala principal y la quadra; y, segundo, su ubicación.

En cuanto al significado de palacio, hay que aclarar que en la documentación medieval toledana se utilizaba para designar la sala principal o salón grande de la casa (Fig.1). Posteriormente, dicho término abarcó un concepto más amplio, refiriéndose a todo el conjunto, pero en origen se refería al salón de la casa principal, salón que reflejaba el poder de una familia o de un oficio importante. Ahora conocemos mejor su origen, heredado de la cultura islámica toledana, así como su transformación y su permanencia en la ciudad bajomedieval ${ }^{1}$.

El otro cuerpo, denominado quadra en los documentos del s. XV, se refiere a una sala de planta cuadrada, que relacionamos con la supervivencia de la qubba islámica, a modo de transmisión o de imitación (fig. 1).

Por lo que se refiere a la ubicación de estos dos cuerpos mayores que constituyen el palacio, o conjunto palaciego, habíamos analizado un aspecto, todavía no muy desa-

1 J. PASSINI, "Los palacios en las casas medievales de Toledo a final de la Edad Media", La Ciudad Medieval de Toledo: Historia, Arqueología y Rehabilitación de la Casa. El Edificio Madre de Dios: Universidad de Castilla-La Mancha, Toledo, 2007, pp. 75-92. 


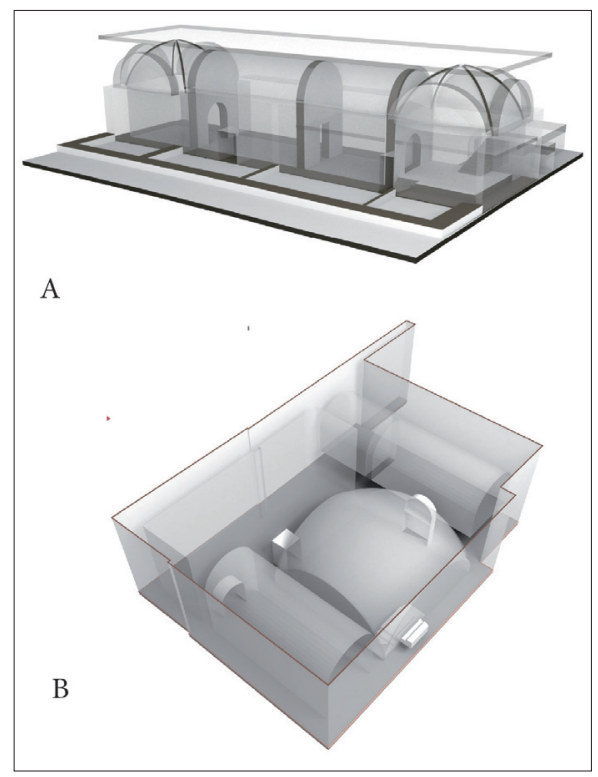

Fig. 1. A- Palacio de don Manuel: estructura en semisótano que retoma el concepto de "palacio" antiguo. B- Estructura en semisótano que retoma el concepto de "quadra". (Dibujo de Matías Capuchino).

rrollado en los palacios mudéjares toledanos, como es el de la implantación física del edificio en relación con el espacio que lo rodea. Cómo se van a cuidar las vistas desde las habitaciones, tanto hacia el interior de la casa como al exterior, construyendo cuerpos en altura para tener una amplia vista sobre la ciudad; patios altos y terrazas sobre plataformas artificiales que se apoyan en sótanos abovedados ${ }^{2}$. Esta novedosa creación de patios altos con vistas es clave para entender la evolución del conjunto palaciego en relación con la ciudad.

Otro objetivo de nuestro trabajo era entender la evolución arquitectónica y espacial del palacio urbano en relación con la ciudad como expresión del poder. Definir lo que quedaba de estos palacios: su plano, su distribución, así como su evolución en los modos de construcción, donde se observa la introducción cada vez mayor de la piedra tallada en sustitución del ladrillo.

La expresión del poder a través de un edificio como el palacio es patente en la importancia del conjunto y en su impacto sobre el tejido preexistente. De tal manera que hemos podido identificar la creación de una plaza delante de un palacio, así como el desarrollo de los balcones, documentados en el s. XV; de la galería, con las vistas que proporcionaba sobre la ciudad; y la creación de una fachada real a la calle. Asistimos al momento en el que se dota a las casas principales de una fachada y de una puerta

2 J. PASSINI, "Los Palacios urbanos medievales en Toledo", La Ciudad Medieval: De la Casa Principal al Palacio Urbano, Toledo, 2011, pp. 219-240. 
principal que se abría a una plaza, con el fin de aumentar la importancia que se daba a la apariencia del edificio y a la relación del interior con el exterior.

Crear una nueva imagen del poder en un tejido urbano en el que todavía persistía una fuerte impronta islámica implicaba sacar al exterior lo que hasta entonces estaba oculto, protegido. Esta apertura se materializó en la creación de una fachada (que antes no existía), de plazas, de balcones a la plaza, de torreones de esquina y de nuevas salas dentro del palacio; así como en la relación entre la escalera y el torreón, la expresión del poder a través de la puerta principal y la creación de los patios altos que abrían visualmente el palacio al exterior. Todas estas estructuras se irán poco a poco introduciendo en las casas de la ciudad a imitación de los nuevos modelos de palacio $^{3}$.

No podemos olvidar la importancia arquitectónica de otra gran obra que en esos momentos se estaba erigiendo en la ciudad: la Catedral y de qué modo el nuevo vocabulario arquitectónico que en ella iba apareciendo influía en otras obras. Un modelo vivo, el del palacio, tomará elementos de otro modelo vivo como era la Catedral.

Para ilustrar nuestras palabras, hemos elegido dos palacios urbanos edificados por poderosos personajes que ponen de manifiesto la importancia de la creación de un nuevo modelo de palacio urbano y que, probablemente, tuvieron un papel destacado en la fijación del concepto de palacio desde la segunda mitad del siglo XIII, el primero, y a principios del s. XV, el segundo. Son el Palacio de don Manuel, actual Convento de Santo Domingo el Antiguo, y el Palacio de Fuensalida.

El primero, el Palacio de don Manuel, todavía de difícil de acceso ${ }^{4}$, ha sufrido diversas modificaciones que encierran, en varias ampliaciones, la fase medieval. Participa en la emergencia de un modelo arquitectónico a partir del arquetipo del palacio islámico, de salón alargado con dos alhanías, que caracteriza el palacio urbano gótico mudéjar a final del siglo XIII. El segundo, el palacio de Fuensalida, introdujo en la primera mitad del s. XV las primicias de un nuevo desarrollo de la casa palaciega a lo largo del Renacimiento.

\section{EI Palacio de don Manuel}

En el último cuarto del s. XIII, alrededor de 1280, el infante don Manuel, hermano del rey Alfonso X, hizo construir un palacio en la ciudad de Toledo que su hijo don Juan Manuel donó, en la primera mitad del s. XIV, al convento de Santo Domingo el Antiguo ${ }^{5}$.

3 La toma de posesión de una casa se hacía siempre por su puerta principal. J. PASSINI, "El portal de las casas principales de los Oter de Lobos (Tordelobos): Aportaciones de los textos", La Ciudad Medieval de Toledo: Historia, Arqueología y Rehabilitación de la Casa. El Edificio Madre de Dios: Universidad de Castilla-La Mancha, Toledo, 2007, pp. 367-378.

4 Muchos de estos Palacios han desaparecido y otros están en manos privadas, lo que dificulta su estudio.

5 J. PASSINI, Casas y casas principales urbanas. El espacio doméstico de Toledo a finales de la Edad Media, Toledo, 2004, p. 87. 


\section{Situación}

Este palacio fue edificado sobre las laderas rocosas norte y oeste de la colina de San Román, en una fuerte pendiente. Ocupaba una manzana entera cerca del convento de Santo Domingo el Antiguo del que le separaba una calle (Fig. 2). Palacio y calles quedaron incluidos en dicho convento en el s. XIV. A pesar de que el convento, desde entonces hasta principios del s. XXI, ha sufrido diversas transformaciones, todavía se pueden distinguir los elementos principales que componían el palacio medieval ${ }^{6}$. El levantamiento de la planta sótano a media pendiente del conjunto, ha permitido poner de manifiesto gran parte de la planta medieval de este palacio urbano real.

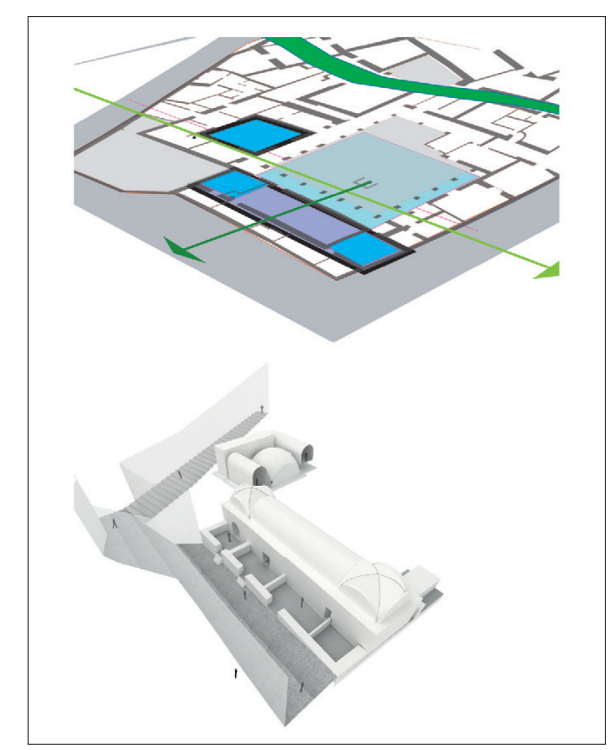

Fig. 2. Sto. Domingo el Antiguo. Planta y 3D del edificio palaciego de don Manuel (dibujo de Matías Capuchino).

\section{Arquitectura}

1. El palacio se componía de dos cuerpos, en forma de L, desarrollados en los dos lados de un patio grande (Fig. 2). Al lado oeste, un gran salón oblongo. Al lado norte, un salón cuadrado que designamos bajo el término de quadra.

2. El nivel inferior copia la estructura formal del nivel superior que se extendía sobre el terraplén.

2.a. Bajo el gran salón oblongo, se extendía una sala abovedada de la misma superficie y de $7 \mathrm{~m}$. de alto, cuyas medidas son 27 x $7 \mathrm{~m}$., incluyendo el espesor de

6 J. PASSINI, op. cit., 2004, p. 89. 
los muros exteriores, de 1,40 m. El cuerpo central de esta sala, cubierta de una bóveda de medio cañón, se extiende sobre un espacio de 4,20 x 13,38 m. Esta sala baja albergaba la caballeriza. En cada extremo, comunica con una pequeña sala, a modo de alhanía, de 4,60 x 4,20 m, cubierta de bóveda de aristas. La situada al sur incluye una escalera larga y ancha que sube desde el nivel de la calle hacia el del patio, situado sobre el terraplén (Fig. 2).

2.b. Al norte, el salón cuadrado, de 7,70 $\mathrm{m}$ de lado, abre en el eje del patio. Se asienta sobre un sótano, de planta cuadrada, abovedado con una cúpula de ladrillo de más de $5 \mathrm{~m}$. de alto, que nace de cuatro arcos laterales de ladrillos (Figs. 1 y 3 ). Este tipo de cúpula se encuentra en varias iglesias mudéjares de Toledo de los s. XIII y XIV, entre las que destaca la iglesia de Santiago del Arrabal.

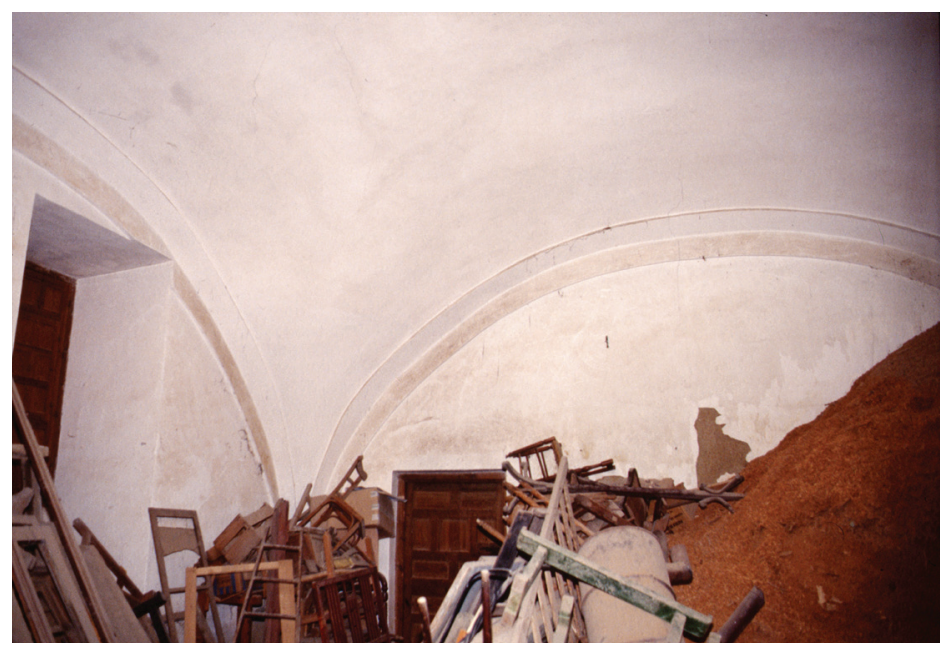

Fig. 3. Sto. Domingo el Antiguo: cúpula bajo la sala cuadrada del conjunto palaciego de don Manuel.

3. Las paredes de los sótanos están hechas de argamasa y encintado de ladrillo. La observación de este aparejo conduce a proponer como fecha probable de edificación el final del s. XIII. Los arcos de herradura apuntada, que se conservan en dos de las esquinas del salón cuadrado al nivel del patio, y los decorados con yeserías en otras partes del convento confirman la influencia de la arquitectura almohade ${ }^{7}$.

En resumen, la persistencia de esas estructuras abovedadas medievales que constituyen la base del Palacio de don Manuel, restituyen una parte de la planta de un gran conjunto palaciego urbano toledano, compuesta de un gran salón oblongo y un salón cuadrado dispuestos en L.

7 Estos arcos fueron descubiertos durante la restauración del convento en la segunda mitad del siglo XX, pero posteriormente fueron tapados. 
El material y el modo de construir perpetúan la tradición islámica. La novedad en este palacio urbano aparece en la edificación de esta casa grande sobre un terraplén amplio extendido en una superficie importante. El sótano refleja el nivel noble de la casa. Tenemos todos los elementos de una arquitectura civil mudéjar heredada de los siglos XII y XIII en Toledo, con el uso del ladrillo para las bóvedas, y de la argamasa y el encintado de ladrillo, de 30 a $40 \mathrm{~cm}$. entre hileras, para los muros.

La ubicación del gran salón y de la quadra ha ofrecido a los constructores la oportunidad de crear un patio alto, con un escape visual lejano. En este palacio, se encuentra también una configuración semejante a la del refectorio del convento de San Clemente donde recientemente se han descubierto los huecos de una arcada del s. XIII abierta hacia el oeste ${ }^{8}$.

\section{El palacio de Fuensalida ${ }^{9}$}

El palacio señorial urbano de Fuensalida se menciona por primera vez en septiembre de 1435 con ocasión de la fundación del mayorazgo de Fuensalida como "las nuestras casas mayores de nuestra morada..." ${ }^{10}$. Fue edificado durante la primera mitad del s. XV por Pedro López de Ayala, Alcalde mayor de Toledo y su mujer doña Elvira de Castañeda.

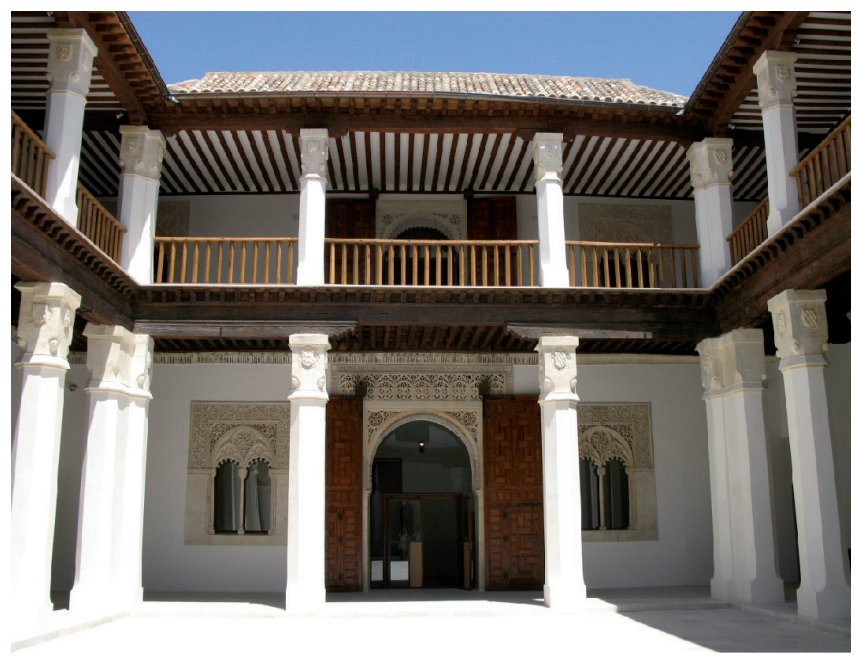

Fig. 4. Palacio de Fuensalida: patio alto, portal y corredores.

8 Véase S. RODRÍGUEZ UNTORIA y J. FERNÁNDEZ DEL CERRO, "De casa a convento: El Monasterio de San Clemente de Toledo", La Ciudad Medieval, Toledo, 2011, pp. 331-364. Una arquería bellamente decorada ha sido también descubierta recientemente en la panda norte del claustro del convento de Santa Fe: F. MONZÓN MOYA, "El antiguo convento de Santa Fe: la desmembración del aula regula islámica y su transformación en un cenobio cristiano", La Ciudad Medieval, Toledo, 2011, pp. 241-276.

9 J. PASSINI, op. cit. 2004, pp. 102-104.

10 Archivo Ducal de Frías leg. 237/11, J. PASSINI, op. cit., 2004, pp. 270. 


\section{Situación}

Las casas mayores de Pedro López de Ayala se sitúan “... a la colaçión de Santo Tomé, çerca de la dicha eglesia”. Se tenían de una parte “... con casas bodegas de Pedro López de Dávalos", “... de la otra parte con la calle e plaza que esta delante la puerta mayor de las dichas casas..." (Fig. 5).

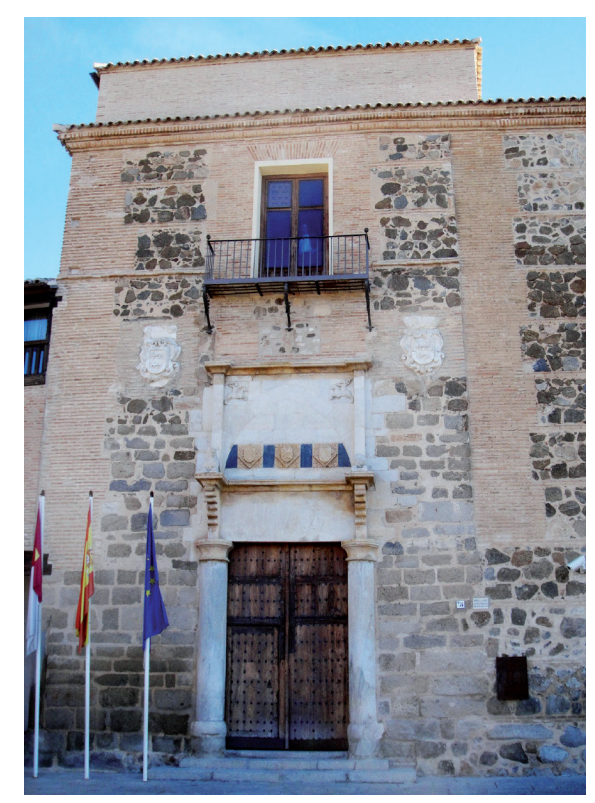

Fig. 5. Palacio de Fuensalida: puerta principal en el torreón restituido en la última restauración.

\section{Arquitectura}

De la arquitectura de este palacio, descrita detalladamente en otra ocasión ${ }^{11}$, destacamos los siguientes puntos:

1. Una superficie sobre el suelo de la totalidad del palacio que llega aproximadamente a $1.400 \mathrm{~m}^{2}$.

2. Un plano en forma de L: al norte y al este dos grandes salones ortogonales; al sur, un portal delimita un patio de planta rectangular.

11 J. PASSINI, op. cit., 2004, pp. 102-103; y P. NAVASCUÉS PALACIO, "Tipología de la casa toledana en el Renacimiento", La maison de la ville à la Renaissance Recherches sur l'habitat urbain en Europe aux XVe et XVIe siècles, Paris, 1983, pp. 77-84 y pp. 177-182. 
3. Al lado del patio dos niveles superpuestos de salones: el nivel inferior abre a un gran pórtico de pilastras, llamado "portal"; el segundo nivel, encima del patio, abre a un corredor (Fig. 4).

4. Un eje visual norte-sur dirigido al campo que cruza el patio a través de una arcada, hoy ocupada por dos ventanas en forma de logia.

5. Dos torreones, simétricos y macizos, de planta cuadrada, de 7,99 x 7,59 m. flanqueaban la fachada del palacio. La torre izquierda, hecha en piedra tallada, acogía la monumental puerta principal (Fig. 5) y albergaba una escalera que subía al patio. La torre derecha incluye, en su nivel inferior la entrada de las caballerizas; en el primer nivel superior, un gran salón cuadrado que da al patio.

6. Unas caballerizas de planta cuadrada, abovedadas "en rincón de claustro", seguidas de otra sala rectangular con bóveda de medio cañón.

\section{Interpretación}

La puerta principal de tipo monumental del palacio de Fuensalida se abre en una esquina de la fachada principal, situación que ocupan también las puertas principales (Fig. 5) de los palacios de Suer Téllez de Meneses (alrededor de 1354), y de don Pedro $(1405)^{12}$. En las dos esquinas de la fachada principal, se observan dos torres de planta cuadrada, independientes de los dos ejes principales del patio, una ocupada por una escalera monumental que sube hacia el patio y, la otra, en la que se superponen a la entrada de la caballeriza, dos niveles de salones cuadrados. La presencia de estas dos torres manifiesta una diferencia importante entre el palacio de Fuensalida y el palacio de don Manuel, diferencia que trataremos más adelante.

\section{Reflexión}

La sala oblonga bajo el Palacio de don Manuel ha servido de arquetipo a la nueva construcción abierta hacia el exterior que corresponde al cuerpo de fachada con el que se amplía el palacio de Fuensalida, mientras que los dos torreones ocupan los extremos, en origen, el lugar de las dos alhanías de planta cuadrada.

En el palacio de don Manuel, el salón largo con sus dos alcobas, todo abovedado y abierto a la calle, era la caballeriza; en uno de los lados, la sala de planta cuadrada alberga una escalera de servicio que sube al patio alto (Fig. 1). En el palacio de Fuensalida, se retoma la misma composición en tres partes, los dos cuerpos extremos, de planta cuadrada tipo alhanía, se transforman en dos torreones cuadrados, uno como entrada y escalera monumental que sube al patio, y toma la forma de una coracha en el acceso al palacio. Al otro extremo, el segundo torreón acoge la entrada a las caballerizas y dos salas cuadradas superpuestas. En ambos casos, el cuerpo central está ocupado por la caballeriza sobre la que se levanta una galería abierta, o sala con vistas al exterior.

12 J. PASSINI, op. cit., 2004, pp. 368-370. 
Podemos ver en esta transformación la aparición de unas nuevas formas arquitectónicas dentro de una continuidad, es decir, manteniendo el respeto a un arquetipo de palacio islámico, estas estructuras anteriores evolucionaron cambiando de función y de significado. El poder se expresaba mediante la creación de una fachada y de una puerta monumental, que daban visibilidad exterior al palacio, a la vez que se abría una plaza delante; y de una escalera monumental en el interior de la entrada (Fig.5). Posteriormente se fueron fijando los elementos de la fachada, pero en estos momentos el palacio se presentaba a través de su fachada y sus estructuras como alegoría del poder, y con el doble fin de ver y ser visto en la ciudad. Poder que también se manifestaba en el uso de la piedra que, en la construcción civil, imitando la obra catedralicia, será símbolo de eternidad. Sin olvidar la recuperación de los elementos en ladrillo de la arquitectura mudéjar y su adaptación a la piedra tallada que observamos en puertas, escaleras y pórticos.

En el palacio de Fuensalida podemos identificar los elementos relacionados con la casa mudéjar, que hereda de manera directa, pero también la adaptación al nuevo concepto de palacio, con un cuerpo de fachada que se desarrollará a lo largo del Renacimiento.

\section{Innovaciones. Relación con el espacio de la ciudad}

Frente al palacio de Fuensalida se abrió una plaza para dar una visión exterior del edificio y que de esta forma tuviera presencia en la ciudad, se pudiera ver. Este espacio frente a la fachada es una intervención novedosa en el tejido de la ciudad medieval, un nuevo concepto arquitectónico con gran repercusión en la trama urbana. Esta transformación del tejido urbano también estaba desarrollándose en torno a la Catedral que, hasta entonces, se encontraba ceñida por edificaciones diversas ${ }^{13}$. Elementos como las loggias o terrazas de los patios altos, utilizadas para ver y ser vistos desde los Palacios durante los actos civiles o religiosos como las procesiones, los encontraremos también en el edificio catedralicio. Otra de las transformaciones que se llevó a cabo fue el desplazamiento de estos conjuntos palaciegos. Mientras que en la ciudad islámica las casas principales ocupaban el centro de la manzana y se cerraban, con discretas vistas al exterior (a lo que contribuía la colocación de las diferentes puertas a distintos lados en función del desnivel), en estos momentos la situación de la casa se desplazó del centro, abriéndose entonces una fachada hacia el exterior, a la ciudad. Además, se colocaron en el mismo plano los establos y la puerta principal, destacada novedad que configurará el frente del palacio. Todos estos elementos se han podido identificar claramente en el palacio de Fuensalida.

\section{Transformación y recuperación del tipo de planta}

La planta del palacio, con dos salas en forma de L, se mantuvo, pero observamos en el palacio de Fuensalida una estructura con dos niveles de salones superpuestos que

13 Durante el siglo XIV se compraron casas para derribarlas y abrir plazas nuevas frente a las puertas de la Catedral. A.M. YUSTE GALÁN, "Puertas y Torres", La Catedral Primada de Toledo. Dieciocho siglos de Historia, Burgos, 2010, pp. 162-179. 
servía para introducir el pórtico que rodea el patio y el corredor de los salones altos, a modo de claustro alto.

El espacio de la casa patio cambia. La morfología general de los salones se transforma, serán más bajos y anchos, con puertas que abrirán hacia el exterior en el portal periférico, en lugar de abrir directamente hacia el interior de un patio. Se abandona la suntuosidad decorativa de la fachada de los salones, desapareciendo, poco a poco las yeserías de sus puertas principales. La sobriedad de la entrada a los salones daba mayor amplitud al pórtico, permitiendo de esta forma que se desarrollase una visión global del patio, con sus pilastras y capiteles. Sin embargo, se mantienen los tramos más largos del portal, de manera simétrica, al nivel de la puerta de los salones. En Toledo se conservan varios ejemplos de este tipo de palacio del s. XV con pórtico grande: lo vemos reflejado en el plano de la Casa de la Inquisición, en el convento de Santa Úrsula o en el palacio de Inés de Ayala (llamado de don Pedro).

Otro elemento importante fue la recuperación de esa arquitectura de ladrillo por parte de la arquitectura de piedra que los pedreros, llegados para trabajar en la Catedral, traspasaron a los palacios, como el gran portal mudéjar del palacio de Oter de Lobos de principios del s. XIV ${ }^{14}$. En un siglo hemos pasado del ladrillo a la piedra tallada: como en la construcción de piedra del portal del patio del Palacio de la Moneda, s. XV, gótico mudéjar, o las grandes portadas de piedra del palacio de Suer Téllez.

La principal novedad que introducirá el palacio de Fuensalida es, por tanto, el cuerpo mayor de la fachada delantera del palacio, un crecimiento que viene a ocupar un estrecho espacio de casas y tiendas. Sin olvidar la tendencia a la desaparición, simultáneamente, del gran salón cuadrado central, observada en el palacio de don Manuel, y de las alcobas de los salones de los s. XII y XIII que observamos en varios palacios. En el de Fuensalida esta sala cuadrada ha perdido su posición central y axial, para desplazarse a la planta del patio alto, en uno de los dos torreones de fachada.

En resumen, las novedades introducidas son:

1. La escalera que ocupa todo el espacio interior de uno de los torreones situado en la esquina, y que tiene dos funciones: la de entrada, como puerta monumental que da a la calle, donde se concentra la decoración heráldica que legitima el poder de la familia y, dentro, otra puerta que da acceso al nivel alto del patio, también monumental, pero menos decorada. En el Palacio de Suer Téllez de Meneses, los escudos de la familia se repiten encima de la puerta alta (entrada al patio). La segunda función de la entrada con una escalera monumental, representa en el palacio un espacio bajo control, asimilable a un espacio fortificado de acceso al patio alto del palacio y que, como una barbacana, sirve de filtro y protección. Este tipo de escalera monumental se está construyendo también en el s. XV en la Catedral para comunicar el claustro bajo con el alto: la llamada Escalera de Tenorio. Escaleras levantadas en piedra de largos y anchos sillares y con pequeñas ventanas de vigilancia en los muros. El modelo también lo tenemos en el Palacio de Suer Téllez de Meneses.

14 J. PASSINI, "El portal de las casas principales de los Oter de Lobos (Tordelobos): Aportaciones de los textos, II Curso de Historia y Urbanismo Medieval", La Ciudad Medieval de Toledo: Historia, Arqueología y Rehabilitación de la casa, El edificio Madre de Dios: Universidad de Castilla-La Mancha", Toledo, 2007, pp. 367-378. 
2. Asociado a la fachada, aparece el espacio de los establos cubierto con bóveda de medio cañón. Sobre ellos se construye la terraza, o portal abierto hacia el exterior del patio alto (Fuensalida). Y, a la derecha, en la otra esquina de la fachada, la entrada lateral a los establos y acceso a la sala primera, la quadra, donde desmontaban los caballeros y que ocupa, en el arquetipo, la alcoba del salón oblongo. Sobre ella, otra sala cuadrada, que en la planta tradicional de una casa islámica sería la alhanía de un gran salón, como en el palacio de Suer Téllez de Meneses. La sala que se correspondería con el gran salón cuadrado, antes centrado en la planta como en el palacio de don Manuel, en este palacio ocupa la planta superior de la entrada a los establos.

\section{Tipología de Bóvedas en los sótanos}

Estos palacios urbanos toledanos se caracterizan también por la utilización de bóvedas en las estructuras de contención sobre las que se construyen. Hemos podido identificar tres tipos diferentes de bóvedas en dichas estructuras, con tres aparejos que merecen toda nuestra atención. Estas bóvedas que cubren las salas cuadradas, o las alhanías son:

1. La cúpula que termina en forma redonda en el Palacio de don Manuel (Figs. 1 y 3), y en el de Jesús y María.

2. La cúpula formada por cuatro bóvedas de aristas, como la del Palacio de Fuensalida.

3. La cúpula octogonal, como la del Palacio de Samuel Leví (actual Museo del Greco), asociada claramente en Toledo, según nuestras observaciones, a obras judías.

Estas tres maneras de construir parecen tener un origen tradicional en relación con las culturas que conviven, como hemos demostrado en el caso de la judería. De los tres tipos, solo queda a finales del s. XV el que se usa en el Palacio de Fuensalida.

\section{Conclusión}

Los dos Palacios que acabamos de evocar manifiestan la voluntad de dos poderosos personajes de la época. La Casa Palacio de don Manuel, hermano del rey de Castilla y León, Alfonso X el Sabio; y el palacio de Fuensalida, del futuro conde de Fuensalida, en aquel momento Alcalde Mayor de la ciudad de Toledo.

El Palacio urbano de la ciudad de Toledo muestra en su desarrollo la evolución de la expresión del poder, afirmando a través de la arquitectura en piedra los nuevos conceptos renacentistas. En la época mudéjar, el poder todavía no se mostraba en el exterior del edificio, concepto que cambiará totalmente a finales del s. XV. El estudio de varios palacios revela cómo se ha constituido este nuevo modelo en el que el palacio de Fuensalida representa el punto de inflexión en el origen de la arquitectura renacentista. 